UCRL-JC-120189

PREPRINT

\title{
Diffractive Coherence in Multilayer Dielectric Gratings
}

B. W. Shore, M. D. Feit, M. D. Perry,

R. D. Boyd, J. A. Britten, and L. Li

This paper was prepared for submittal to the Seventh Rochester Conference on Coherence \& Quantum Optics Rochester, New York

June 7-10, 1995

May 26, 1995

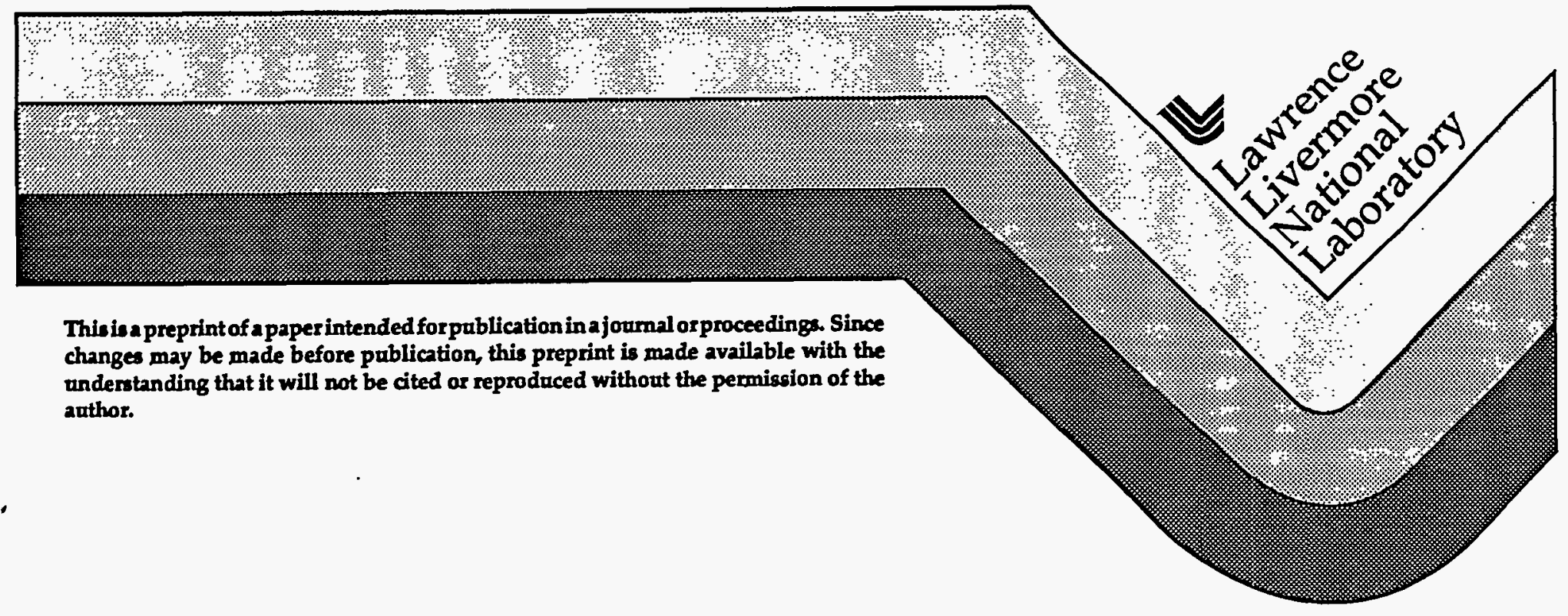




\section{DISCLAIMER}

This document was prepared as an account of work sponsored by an agency of the United States Government. Neither the United States Government nor the University of California nor any of their employees, makes any warranty, express or implied, or assumes any legal liability or responsibility for the accuracy, completeness, or usefulness of any information, apparatus, product, or process disclosed, or represents that its use would not infringe privately owned rights. Reference herein to any specific commercial product, process, or service by trade name, trademark, manufacturer, or otherwise, does not necessarily constitute or imply its endorsement, recommendation, or favoring by the United States Government or the University of California. The views and opinions of authors expressed herein do not necessarily state or reflect those of the United States Government or the University of California, and shall not be used for advertising or product endorsement purposes. 


\section{DISCLAIMER}

Portions of this document may be illegible in electronic image products. Images are produced from the best available original document. 


\title{
DIFFRACTIVE COHERENCE IN MULTILAYER DIELECTRIC GRATINGS
}

\author{
B. W. Shore, M. D. Feit, M. D. Perry, R. D. Boyd, J. A. Britten \\ Lawrence Livermore National Laboratory, Livermore, CA 94550 \\ and Lifeng. Li \\ Optical Sciences Center, University of Arizona, Tucson AZ 85721
}

\begin{abstract}
Successful operation of large-scale high-power lasers, such as those in use and planned at LLNL and elsewhere, require optical elements that can withstand extremely high fluences without suffering damage. Of particular concern are dielectric diffraction gratings used for beam sampling and pulse compression. Laser induced damage to bulk dielectric material originates with coupling of the electric field of the radiation to bound electrons, proceeding through a succession of mechanisms that couple the electron kinetic energy to lattice energy and ultimately to macroscopic structural changes (e.g. melting). The constructive interference that is responsible for the diffractive behavior of a grating or the reflective properties of a multilayer dielectric stack can enhance the electric field above values that would occur in unstructured homogeneous material. Much work has been done to model damage to bulk matter. The presence of nonuniform electric fields, resulting from diffractive coherence, has the potential to affect damage thresholds and requires more elaborate theory. We shall discuss aspects of work directed towards understanding the influence of dielectric structures upon damage, with particular emphasis on computations and interpretation of electric fields within dielectric gratings and multilayer dielectric stacks, noting particularly the interference effects that occur in these structures.
\end{abstract}

\section{INTRODUCTION}

Interference between coherent portions of electromagnetic waves, to produce stationary standing waves, lead to numerous fascinating effects of practical interest. For the simplest case of a single monochromatic standing plane wave, the electric field at an antinode becomes 2 times the free space value for a traveling plane wave; the corresponding intensity is enhanced by a factor of 4 over that of a plane wave. Such electric field enhancements can increase the possibility of optical damage, through increase of multiphoton ionization or of field-induced electron avalanche. Designers of optical systems intended for use with intense fields must consider carefully the possibility of constructive interference producing small regions where electric fields become excessive.

Interference effects, being inherently wave attributes, have potential to become particularly pronounced in optical elements that rely on diffraction. Foremost amongst these elements are diffraction gratings, now being widely used under conditions of intense fields in chirped pulse amplifiers. Multilayer dielectric stacks, used to create highly reflecting surfaces, also have potential to create appreciable field enhancements. For such applications it becomes important to understand the role of interference, as exhibited by electric field magnitudes. We here present some illustrative examples of electric fields in gratings and multilayer dielectric stacks. 


\section{GRATINGS}

A grating can be idealized as a set of perfectly periodic corrugations. Using a Cartesian coordinate system with y axis vertical, we take the grating surface to be independent of the horizontal direction $\mathrm{z}$ and periodic in the horizontal direction $\mathrm{x}$.

When monochromatic plane-wave radiation impinges on such a grating, and the propagation vector lies in the $x, y$ plane, then the electromagnetic field surrounding the grating is independent of $z$. With this restricted geometry any incident wave can be expressed as a combination of two orthogonal polarizations, TE (electric field parallel to the grooves and perpendicular to the plane of propagation) and TM (magnetic field parallel to the grooves and perpendicular to the plane of propagation). Because field components parallel to a surface must be continuous, the two polarization choices are associated with two distinct boundary continuity conditions for the field: for TE polarization the electric field must be continuous, whereas for TM polarization the electric field may be discontinuous.

\section{DIFFRACTED WAVES}

The electromagnetic field above (or below) a grating consists of assorted diffracted waves, each with a propagation vector in the $x, y$ plane (of incidence). Although the relative strength of the various waves generally depends upon the shape of the grating surface (i.e. whether the grooves are rectangular, triangular, or sinusoidal in cross section), the direction of the orders is fixed by the wavelength of the light $\lambda$, the spacing $d$ between grooves, and the angle of incidence in accord with the grating equation

$$
n^{\prime} \sin \beta_{m}=n \sin \alpha+(m \lambda / d)
$$

where $\alpha$ is the angle of incidence, in a medium with refractive index $n$, and $\beta \mathrm{m}$ is the angle of diffraction of order $m$ (in reflection or transmission), in medium with refractive index $n$. The coherent superposition of these various waves, like the standing waves above a mirror, creates a pattern of nodes and antinodes.

\section{THE INTERFERENCE PATTERN}

Above the grating, the interference between the incident plane wave traveling toward the surface, and the returning diffracted waves, gives rise to a stationary standing wave pattern of nodes and antinodes of the electric field.

Below the grating there is no incident upward wave (apart from possible reflections at an exit interface). Nevertheless, standing wave patterns can be formed by waves traveling right and left. Within the grooves of the grating structure the field may be strongly affected by the form of the grating surface or by eigenmode structures.

\section{THE METALLIC SURFACE}

The simplest interference effect occurs above a mirror. The high electrical conductivity that characterizes a metal forces the electric field to vanish within a very short distance into the metal; for a perfect conductor the electric field has a node along the surface. The reflecting properties of metallic surfaces for coherent monochromatic light can be described as a 
standing wave above the surface. For normal incidence the electric field has a node at the surface, and an antinode one quarter wave above the surface The allowed standing-wave patterns of electric field within an enclosed metallic container have been widely studied for application to microwave devices; many textbooks present pictures of the various nodal patterns.

\section{HOLOGRAPHIC METAL GRATINGS}

Traditional diffraction gratings, widely used in spectrometers to analyze broadband lowintensity light sources, contain corrugated metallic surface of precisely parallel periodic grooves. The metallic surface acts to reflect the light, and the periodic groove structure acts to diffract the light, sending different colors back in different directions. For many decades such gratings were produced either by the engraving action. of an extremely precise mechanical ruling engine, or by replicating a master grating produced by such an engine. Today grating manufacturers often use holographic techniques, relying on the stability of continuous-wave laser wavefronts, rather than mechanical stability of an engine, to provide the precision.

With such holographic approaches a photosensitive surface is exposed to a standing wave pattern created by interfering two highly coherent laser beams. The subsequent development of the latent image of the periodic interference pattern creates a corrugated surface. By coating the grooved surface with a thin metallic film, it is possible to create a highly efficient reflection grating. Alternatively, the pattern can be transferred into a (more robust) underlying dielectric substrate by chemical etching procedures.

A cutaway view of a grating formed by either of these methods reveals a profile that is characteristic of the method. For gratings produced by transfer etching, the profiles typically are trapezoidal or rectangular (lamellar) in cross section. Gratings produced by metallic coating of photoresist are more complicated; they may differ appreciably from the sinusoidal intensity distribution of the latent image.

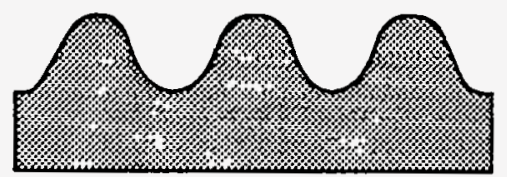

Pattern from photoresist

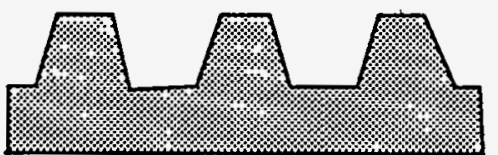

Pattern from etching

\section{HIGH EFFICIENCY METAL GRATINGS}

To maximize the diffracted energy it is customary to use a grating whose period is too small to allow more that two orders, $\mathrm{m}=0$ (specular reflection) and $\mathrm{m}=-1$. Typically such gratings are used near the Littrow angle, such that the diffracted -1 order reflects at the angle of incidence (autocollimation):

$n \sin \alpha=-\frac{\lambda}{2 d}$ 
When only two orders occur then the diffraction efficiency of a metallic grating exhibits an interesting regularity with increasing groove depth: the efficiency varies between near zero (antiblazing) and nearly complete (perfect blazing).

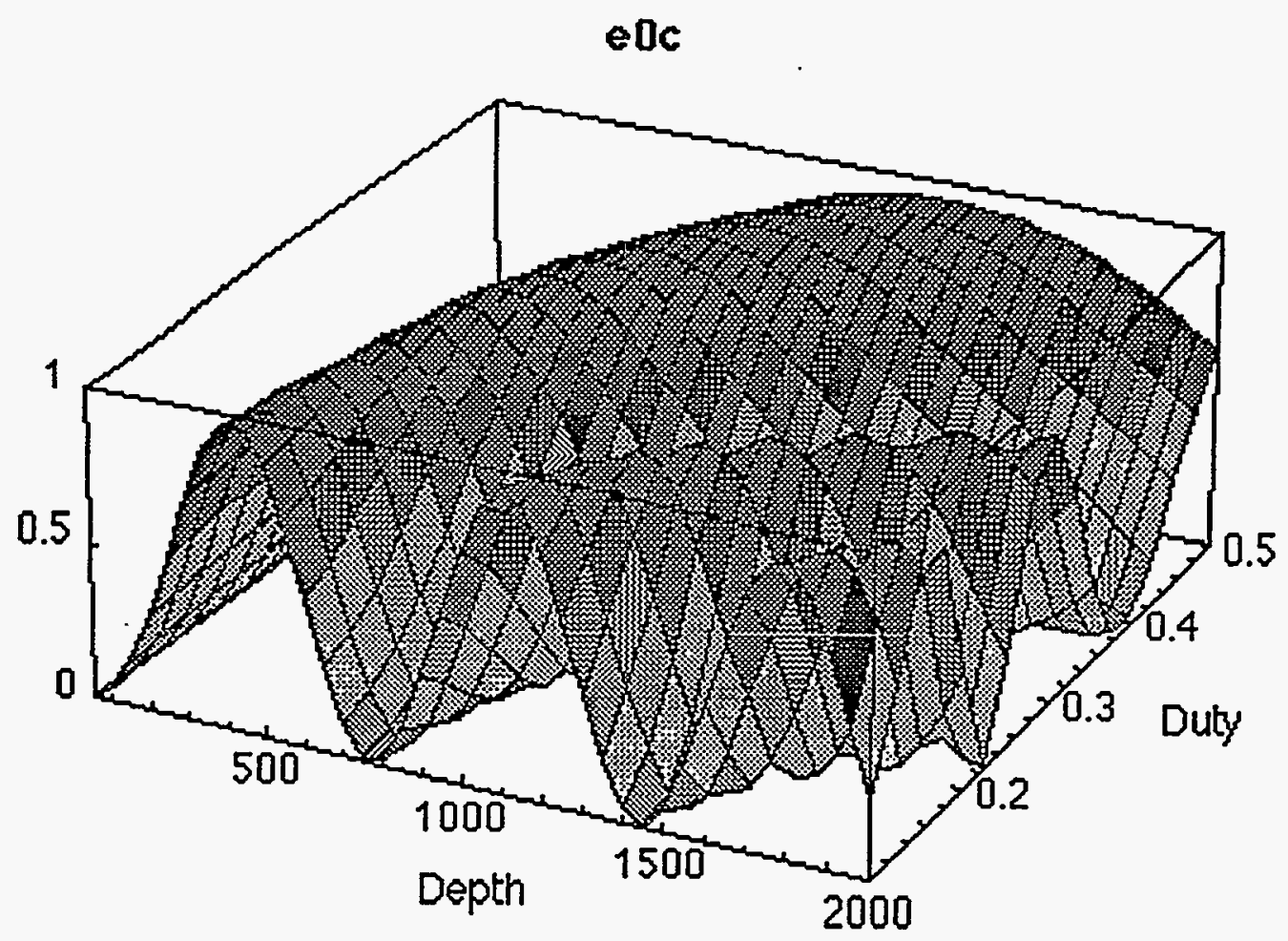

Figure illustrates this periodic dependence for a metallic grating (gold coated photoresist), as a function of groove depth and groove shape (expressed as duty cycle, or fraction of a period occupied by grating ridges) when used at Littrow angle with TE polarization.

\section{TE FIELD ABOVE METAL GRATING}

It now well known that this regular pattern is a manifestation, within the grooves, of the same sort of mode structure found in metallic enclosures and waveguides. By increasing the groove depth we allow standing wave patterns to develop: first a quarter wave, then a half wave, etc. 


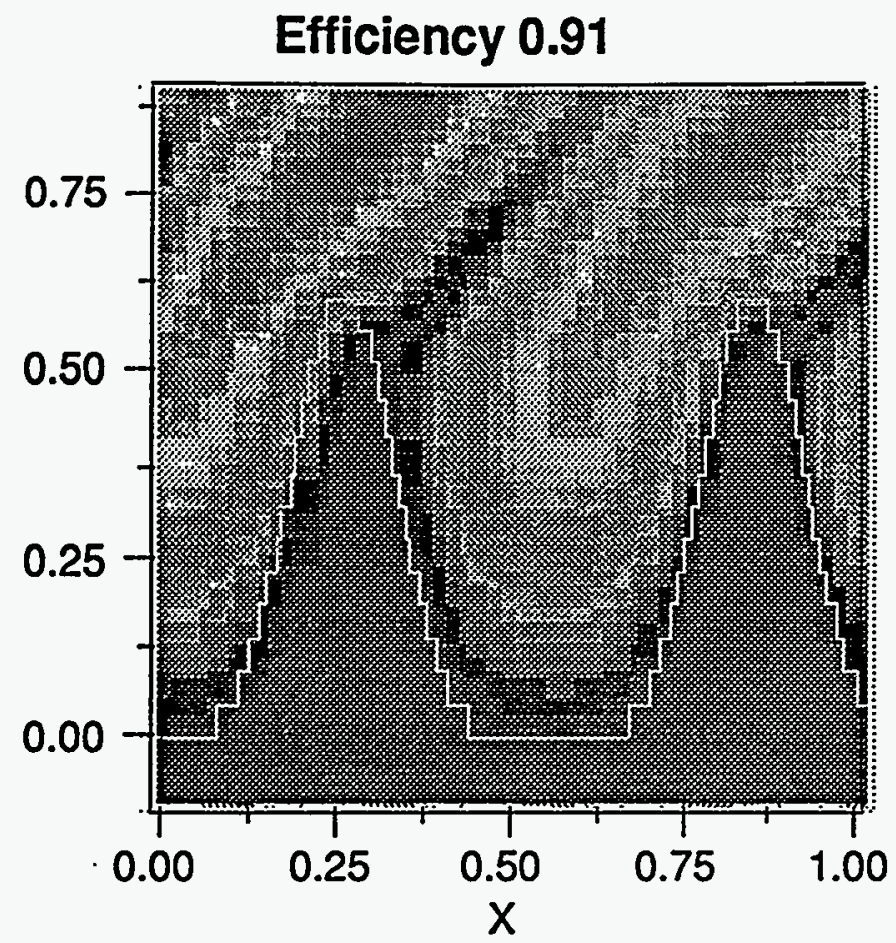

\section{Efficiency 0.001}

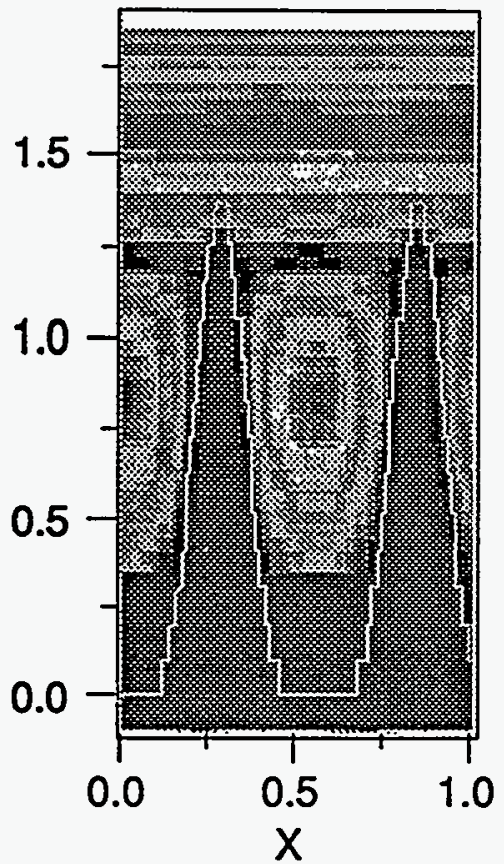

Figure illustrates two examples of these TE fields, for grooves that produce high efficiency and for deeper grooves that produce negligible diffraction (an antiblazed grating).

\section{HIGH EFFICIENCY TM GRATING}

In general, metal gratings are designed for use with TM polarization (magnetic field parallel to the grooves). High efficiencies are obtained with grooves that are relatively shallow (compared with what is required for TE polarization).

Holographically prepared metal gratings used with TM polarization have efficiencies that are relatively insensitive to the grating profile, as expressed by the duty cycle (the fraction of the period which is taken up by the grating ridge). This property has great value during the manufacturing. 


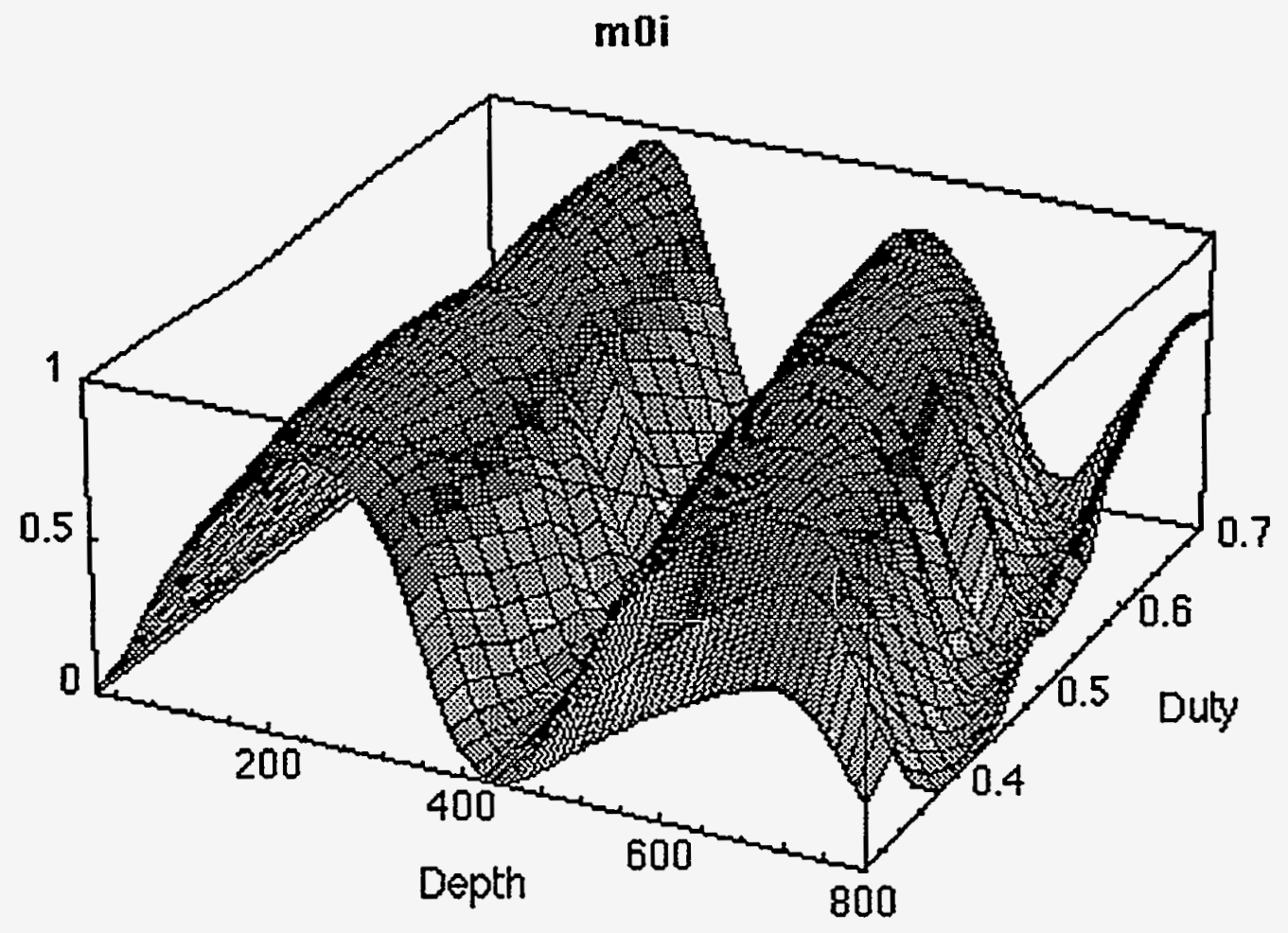

Figure illustrates this periodic dependence for a metallic grating (gold coated photoresist), as a function of groove depth and groove shape (expressed as duty cycle, or fraction of a period occupied by grating ridges) when used at Littrow angle with TM polarization.

\section{TM FIELD DISTRIBUTION}

The electric field of a metal grating used in TM polarization is not required to maintain a node at the grating surface, unlike the electric field with TE polarization.

The electric field for a high efficiency metal grating exhibits a standing wave pattern whose nodes and antinodes extend down to the surface. Such a grating has regions of constructive interference where the electric field is twice the free-space traveling-wave value.

High fields at the surface occur both for high and low efficiency use. 

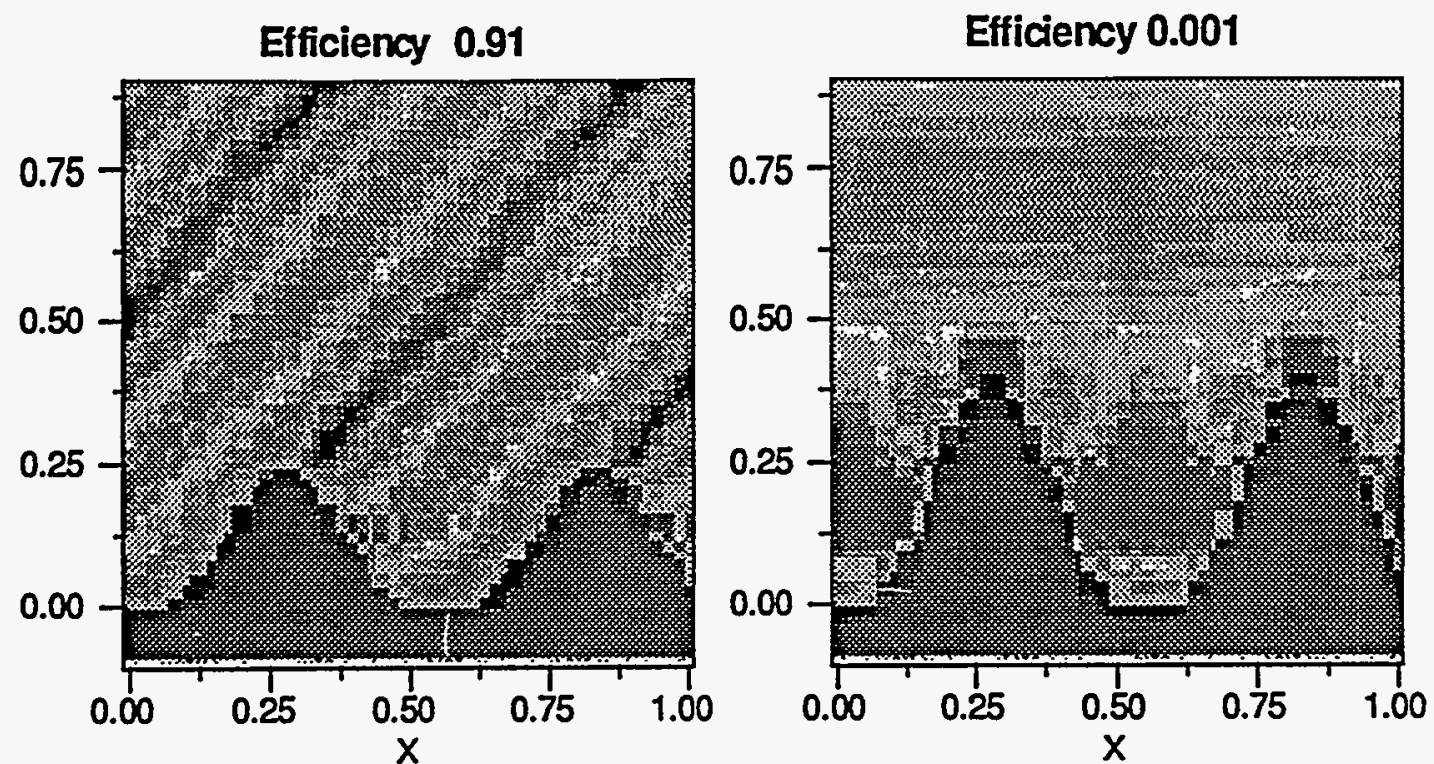

Figure illustrates two examples of TM fields above a near-sinusoidal profile grating, for grooves that produce high efficiency and for deeper grooves that produce negligible diffraction (an antiblazed grating).

\section{THE MULTILAYER DIELECTRIC REFLECTION GRATING}

Metallic gratings must be used in reflection, and (apart from well documented grating "anomalies") have efficiencies that are relatively insensitive to wavelength. Dielectric diffractive structures, by contrast, can be designed to fulfill wavelength sensitive design criteria in both reflection and transmission, as evidenced by the variety of commercial thinfilm dielectric devices used as filters, polarizers, beam splitters, etc.

The combination of a dielectric grating to provide dispersion and a multilayer dielectric stack to provide reflection makes possible the creation of a high efficiency dielectric reflection grating.

A simple design makes use of the properties of quarter wave stacks as highly reflecting optical elements. One creates a succession of thin films, alternately a high refractive index and a low refractive index, each of which introduces a quarter wave phase increment to a wave incident at a specified angle (the Littrow angle for the gratings of interest). Such a stack, combined with a dielectric grating, can produce a grating whose reflection efficiency (in order -1) exceeds $95 \%$. 


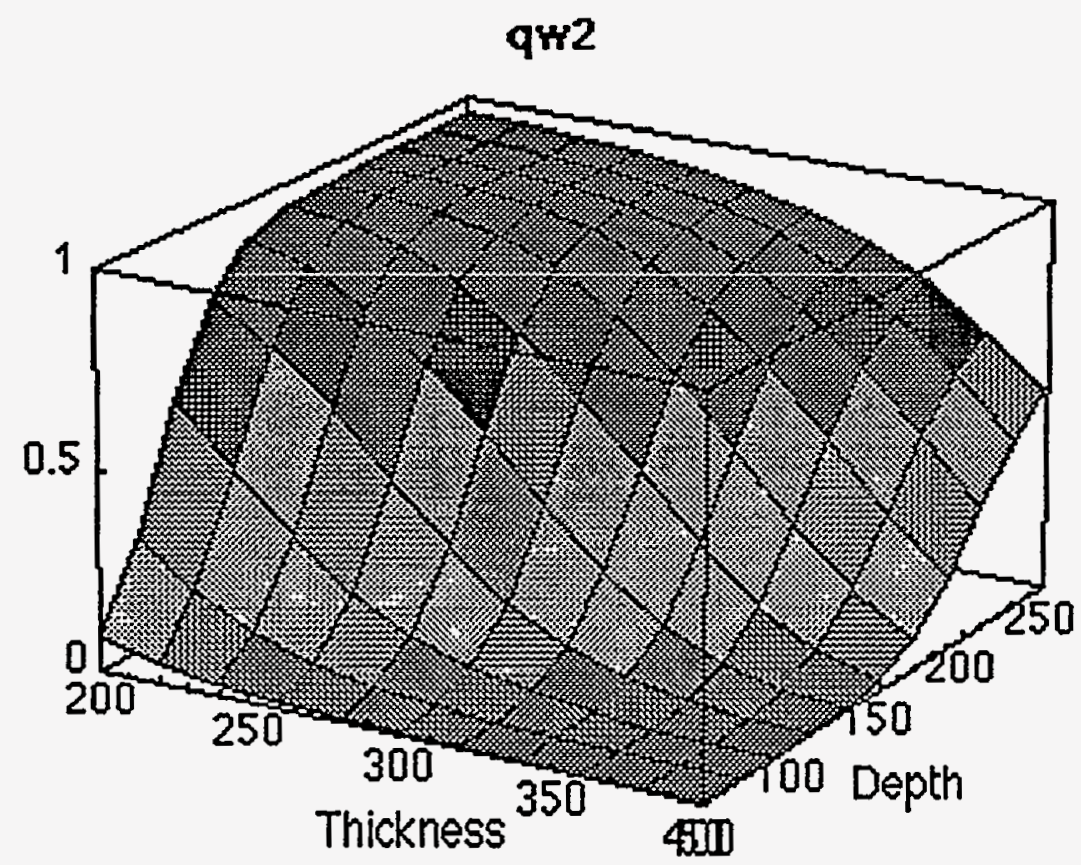

Figure shows the efficiency vs. groove depth and top layer thickness, for a grating with rectangular groove profile (as would be obtained by transfer etching into the surface), illuminated at the Littrow angle by $1053 \mathrm{~nm}$ light, TE polarization. 


\section{ELECTRIC FIELD DISTRIBUTION IN MLD GRATING}

The distribution of electric fields around this structure shows a pattern of standing waves above the surface, as is to be expected from the combination of nearly equal amplitudes of incident and reflected light. Within the grating structure itself, the strength of the electric field exhibits a succession of nodes and of antinodes of diminishing magnitude. These are the result of the interference between upward and downward traveling waves.

There are two regions in which constructive interference is as large as the twofold enhancement of a mirror: at one corner of the (rectangular) grating, and at the first interface where a high-index material overlays a low index material. The high reflectivity of a quarter wave stack accompanies a standing wave pattern whose nodes align with the low-high index interfaces, and whose antinodes align with the high-low index interfaces.

\section{Index}

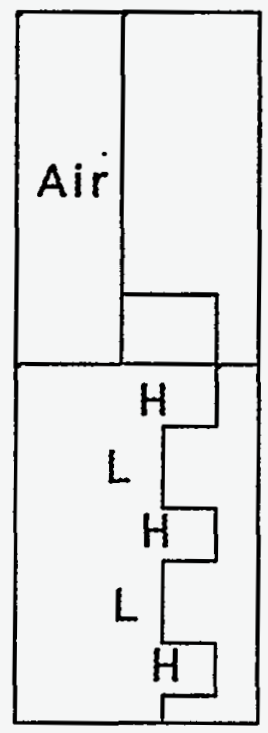

E Field

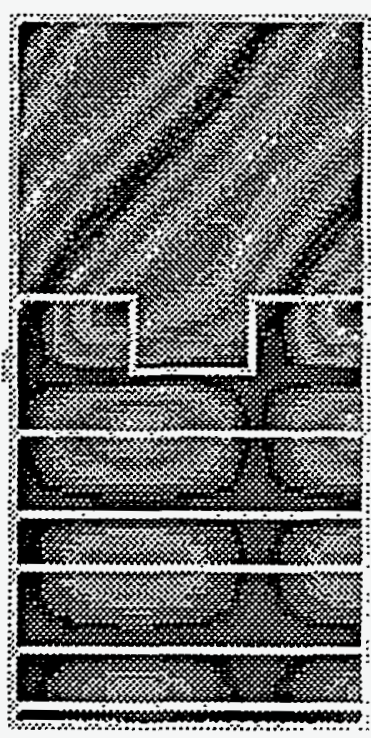

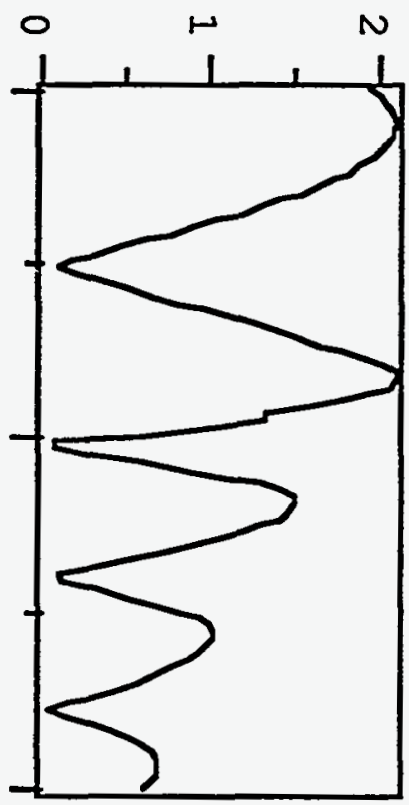

Figure illustrates the electric field magnitude in such a quarter wave stack.

\section{THE MODIFIED HLL STACK}

The interface between different materials is the weakest mechanical region. The presence of high electric fields at interfaces between different material is therefore undesirable. It is customary to modify the quarter wave stack so as to shift the peak fields into regions of low index, where their effects are less than in a high index region

The interfaces of different materials are particularly sensitive to damage by intense fields. It is therefore desirable to shift the peak electric field away from the interface. 
We have developed an alternative design which accomplishes this, while maintaining the desired high reflectivity for use under a grating. The design makes use of two quarter waves of low index material and one quarter wave of high index material, as defined for vertically incident light of wavelength $830 \mathrm{~nm}$. (The use angle is 52 degrees for wavelength $1053 \mathrm{~nm})$.
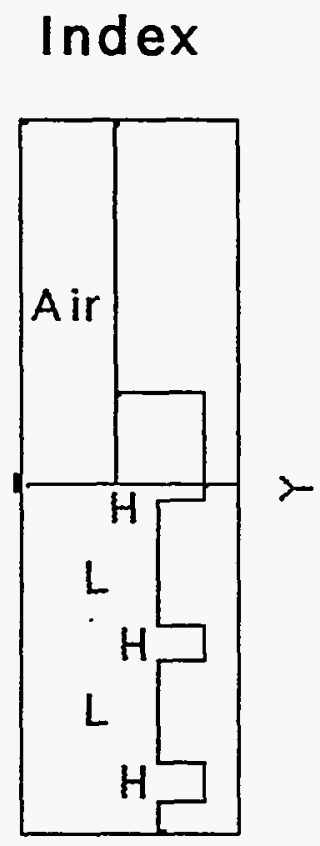

E Field

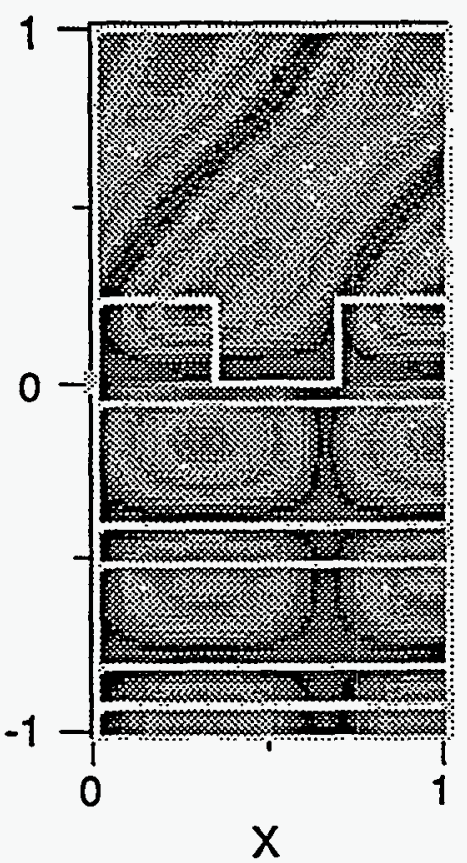

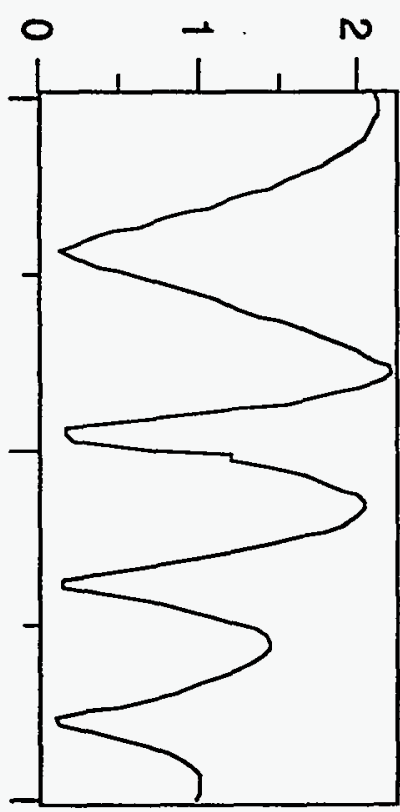

Figure shows the electric field magnitude in a HLL stack

This design shifts the peak field within the multilayer stack into the region of low index. It does not eliminate the high field at the top edge of the grating.

\section{ACKNOWLEDGMENTS}

This work was supported under the auspices of the U.S. Department of Energy at Lawrence Livermore National Laboratory under contract W-7405-Eng-48. 


\section{SUPPLEMENTARY FIGURES}

\section{THE DEEP DIELECTRIC TRANSMISSION GRATING}

The ability of a periodic structure to create multiple diffraction orders depends critically on the difference between the refractive indices of the grating material and the surrounding media. Dielectric gratings are intrinsically poor reflectors. However, they can have very high efficiency in transmission, rivaling the reflection efficiency of a metallic grating. To achieve this goal the groove spacing must, as with a high efficiency metallic grating, be small enough to allow only two orders. Then, as with metallic gratings, the diffraction efficiency oscillates between nearly zero and nearly unity with increasing groove depth. A succession of high efficiency solutions exist, with efficiencies approaching unity.

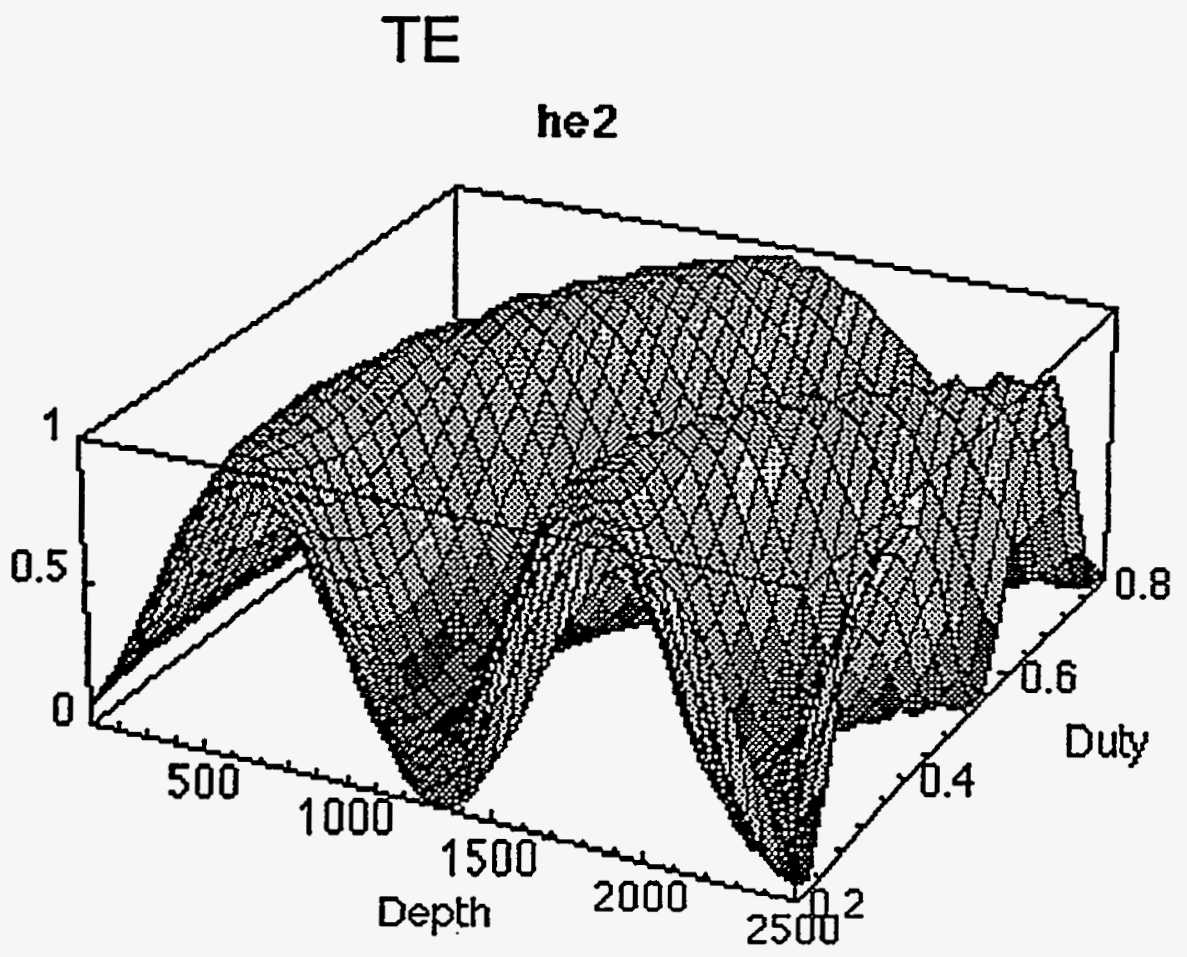

Figure shows an example of efficiency vs depth for a lamellar-profile transmission grating etched in glass. 


\section{TM}

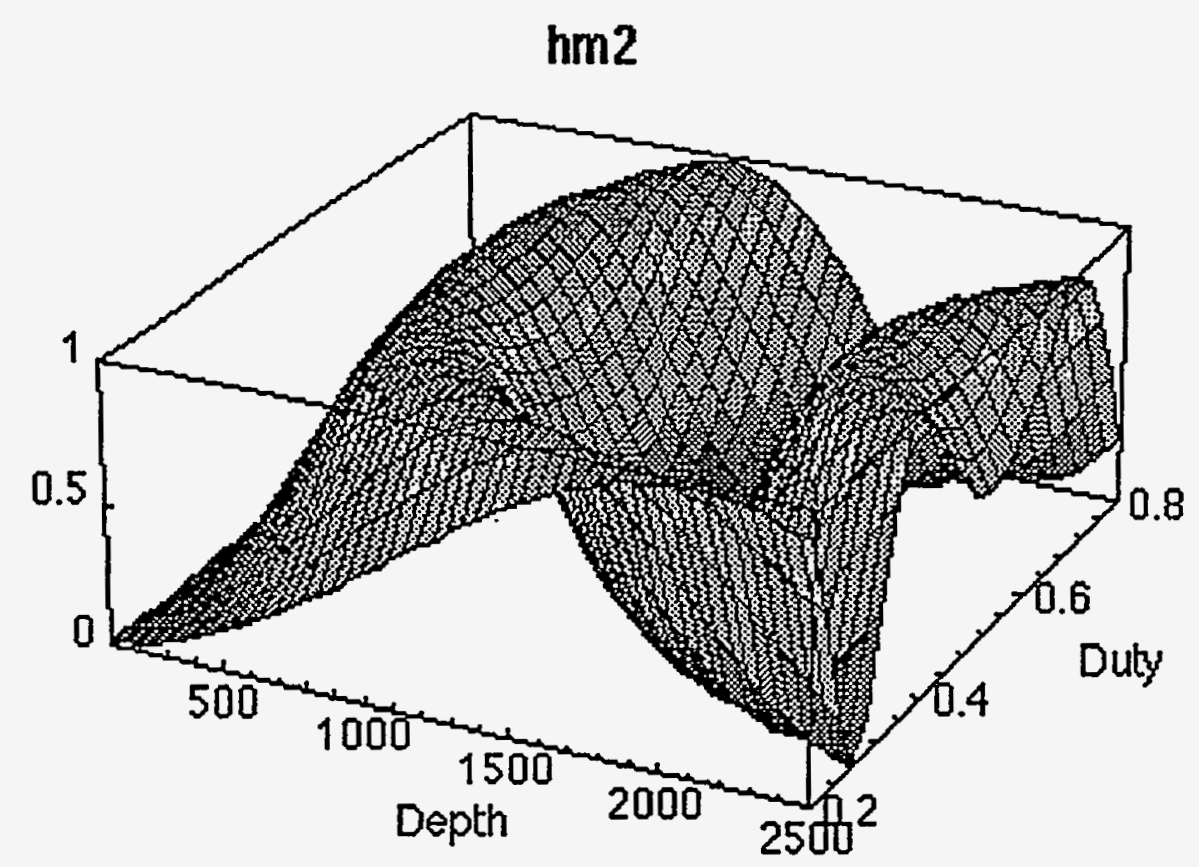

Figure shows an example of efficiency vs depth for a lamellar-profile transmission grating etched in glass. 


\section{FIELDS IN DEEP DIELECTRIC GRATING}

Fixing the groove spacing to permit only two orders permits the design of a high efficiency transmission grating. Such gratings have application in the LLNL laser program as beam steering gratings at the final focus of beam lines directed to targets in the National Ignition Facility (NIF) laser.

Present NIF designs call for the amplification of $1053 \mathrm{~nm}$ light $(1 \omega)$, which will be converted into third harmonic light $(3 \omega)$ in $\mathrm{KDP}$ frequency conversion crystals. A deep dielectric grating will direct the 300 light toward the target while leaving unconverted 100 and 200 light undeflected.

$1 \omega$

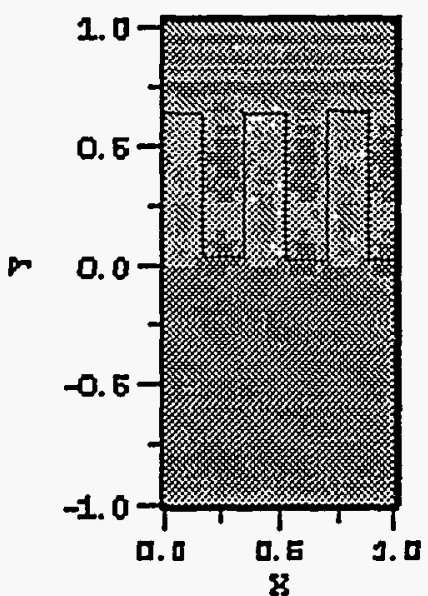

$2 \omega$

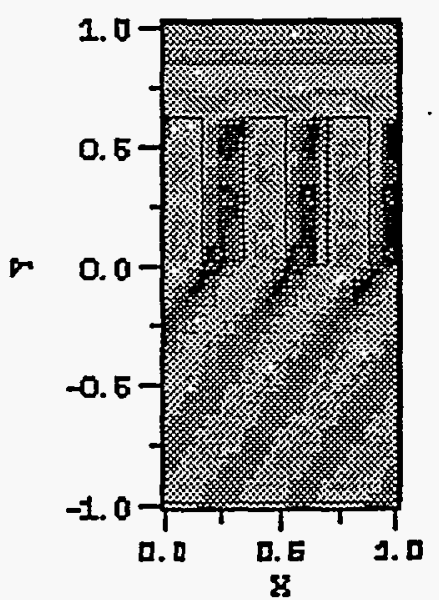

$3 \omega$

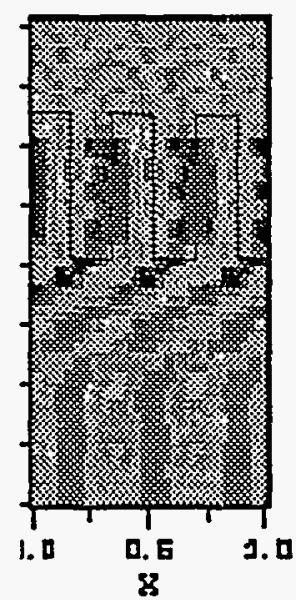

Figure shows the $T E$ electric field, at $1 \omega$ (high efficiency diffraction in transmission), and $2 \omega$ and $3 \omega$ (low efficiency in diffraction), in a deep dielectric transmission grating. 